\title{
Synthesis, Gastroprotective Effect and Cytotoxicity of New Amino Acid Diterpene Monoamides and Diamides ${ }^{\dagger}$
}

\section{Guillermo Schmeda-Hirschmann ${ }^{1}{ }^{\text {, }}$, Mariano Walter Pertino ${ }^{1}$, Jaime A. Rodriguez ${ }^{2}$, Francisco Monsalve ${ }^{3}$, Daniel Droguett ${ }^{4}$ and Cristina Theoduloz ${ }^{2}$}

1 Laboratorio de Química de Productos Naturales, Instituto de Química de Recursos Naturales, Universidad de Talca, Casilla 747, Talca, Chile

2 Laboratorio de Cultivo Celular, Departamento de Bioquímica Clínica, Facultad de Ciencias de la Salud, Universidad de Talca, Talca, Chile

3 Departamento de Ciencias Básicas, Facultad de Ciencias de la Salud, Universidad de Talca, Talca, Chile

4 Departamento de Estomatología, Facultad de Ciencias de la Salud, Universidad de Talca, Talca, Chile

$\dagger$ Dedicated to the memory of Professor Jaime A. Rodriguez

* Author to whom correspondence should be addressed; E-Mail: schmeda@utalca.cl; Tel.: +56-71-200288; Fax: + 56-71-201573.

Received: 9 August 2010; in revised form: 5 October 2010 / Accepted: 14 October 2010 / Published: 21 October 2010

Abstract: Following our studies on the gastroprotective effect and cytotoxicity of terpene derivatives, new amides were prepared from the diterpene 8(17)-labden-15,19-dioic acid (junicedric acid) and its 8(9)-en isomer with $C$-protected amino acids (amino acid esters). The new compounds were evaluated for their gastroprotective effect in the ethanol/HClinduced gastric lesions model in mice, as well as for cytotoxicity using the following human cell lines: normal lung fibroblasts (MRC-5), gastric adenocarcinoma cells (AGS) and liver hepatocellular carcinoma (Hep G2). A dose-response experiment showed that at $25 \mathrm{mg} / \mathrm{kg}$ the C-15 leucyl and C-15,19-dileucylester amides of junicedric acid reduced gastric lesions by about 65.6 and $49.6 \%$, respectively, with an effect comparable to lansoprazole at $20 \mathrm{mg} / \mathrm{kg}$ (79.3\% lesion reduction). The comparison of the gastroprotective effect of 18 new amino acid ester amides was carried out at a single oral dose of $25 \mathrm{mg} / \mathrm{kg}$. Several compounds presented a strong gastroprotective effect, reducing gastric lesions in 
the 70.9-87.8\% range. The diprolyl derivative of junicedric acid, the most active product of this study $(87.8 \%$ lesion reduction at $25 \mathrm{mg} / \mathrm{kg})$ presented a cytotoxicity value comparable with that of the reference compound lansoprazole. The structure-activity relationships are discussed.

Keywords: labdane diterpenes; amino acid ester amides; gastroprotective effect; cytotoxicity

\section{Introduction}

Natural products, including plant drugs, have been used as gastroprotective agents all over the World. The natural products-derived therapeutic agents include the terpenes glycyrrhetic acid and sodium ecabet [1-5]. Several plant drugs are used in Latin American traditional medicine to treat symptoms related with gastric ulcers. The active gastroprotective constituents of some of the crude drugs are terpenes that present relevant activity in animal as well as in in vitro models [6-17].

It has been established that chronic gastric ulcers might lead to gastric and pancreatic cancer $[18,19]$. The resin of the tree Araucaria araucana is used to relieve gastric pain and the gastroprotective effect of the crude drug and main diterpene constituents has been reported [15]. The study started an investigation on the structure-activity relationships of labdane diterpenes from the resin for gastroprotective as well as for cytotoxic effect on normal and cancer cell lines [14,15,20]. Amide derivatives from labdane diterpenes with increased gastroprotective effect and low cytotoxicity were prepared [20]. However, the aromatic amines, used to prepare the amides, do not correspond to usual mammalian metabolites. Therefore, we selected amino acids esters to prepare new amides.

Figure 1. General procedure to prepare C-19 amides from imbricatolic acid and acetoxyimbricatolic acid as well as from the 8(9)-en isomers.

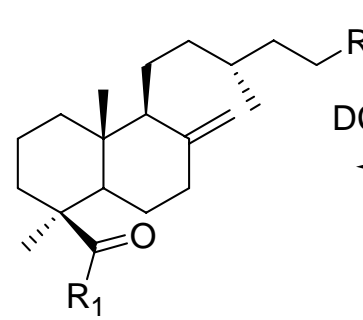

$\mathrm{R}: \mathrm{OH}$ or OAC

$R_{1}$ : AAE

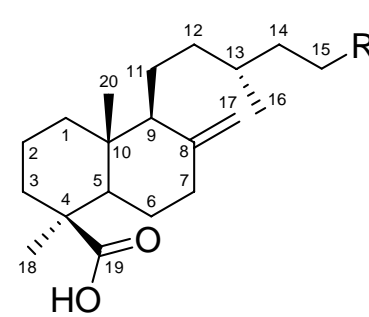

$\mathrm{R}: \mathrm{OH}$ imbricatolic acid

R: OAc acetoxyimbricatolic acid

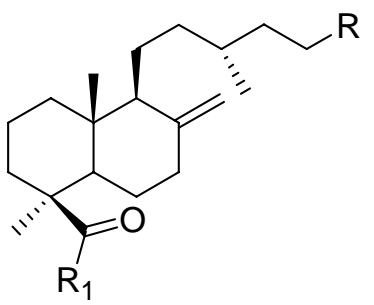

$\mathrm{R}: \mathrm{OH}$ or OAC

$\mathrm{R}_{1}$ : $\mathrm{AAE}$

Reagents and conditions: (a) appropriate amino acid ester (AAE), $N, N^{\prime}$-dicyclohexylcarbodiimide (DCC), dimethylaminopyridine (DMAP), dichloromethane $\left(\mathrm{CH}_{2} \mathrm{Cl}_{2}\right)$; (b) oxalyl chloride $(\mathrm{COCl})_{2}$, $\mathrm{CH}_{2} \mathrm{Cl}_{2}$, AAE.

A previous study on the synthesis of C-19 amino acid ester monoamides was carried out using imbricatolic acid and its acetate, as well as the 8(9)-en isomers, as starting compounds (Figure 1). The new C-19 monoamides showed gastroprotective effects but the activity was lower than that found for 
amides of aromatic amines [21]. There is, however, no information on the gastroprotective effect of labdane diterpene amides bearing the amide at $\mathrm{C}-15$, nor is the effect of $\mathrm{C}-15$, 19-diamides on preventing gastric lesions or modulating cytotoxicity known.

The aim of the present work was to synthesize new diterpene amides starting from the labdane diterpene imbricatolic acid. The starting compound was oxidized to obtain 8(17)-labden-15,19-dioic acid (junicedric acid) and then isomerized to the 8(9)-en derivative to prepare the new compounds. The new amides were assessed for gastroprotective effects in the ethanol/HCl-induced gastric lesions model in mice, as well as for cytotoxicity on normal human lung fibroblasts and the tumor cell lines AGS (gastric adenocarcinoma) and HepG2 (hepatocytes). The amino acids used to prepare the amides were $C$-methyl or ethyl esters of glycine, valine, leucine, proline and tryptophan.

Figure 2. General procedure to prepare monoamides and diamides from dicarboxylic labdane diterpenes.

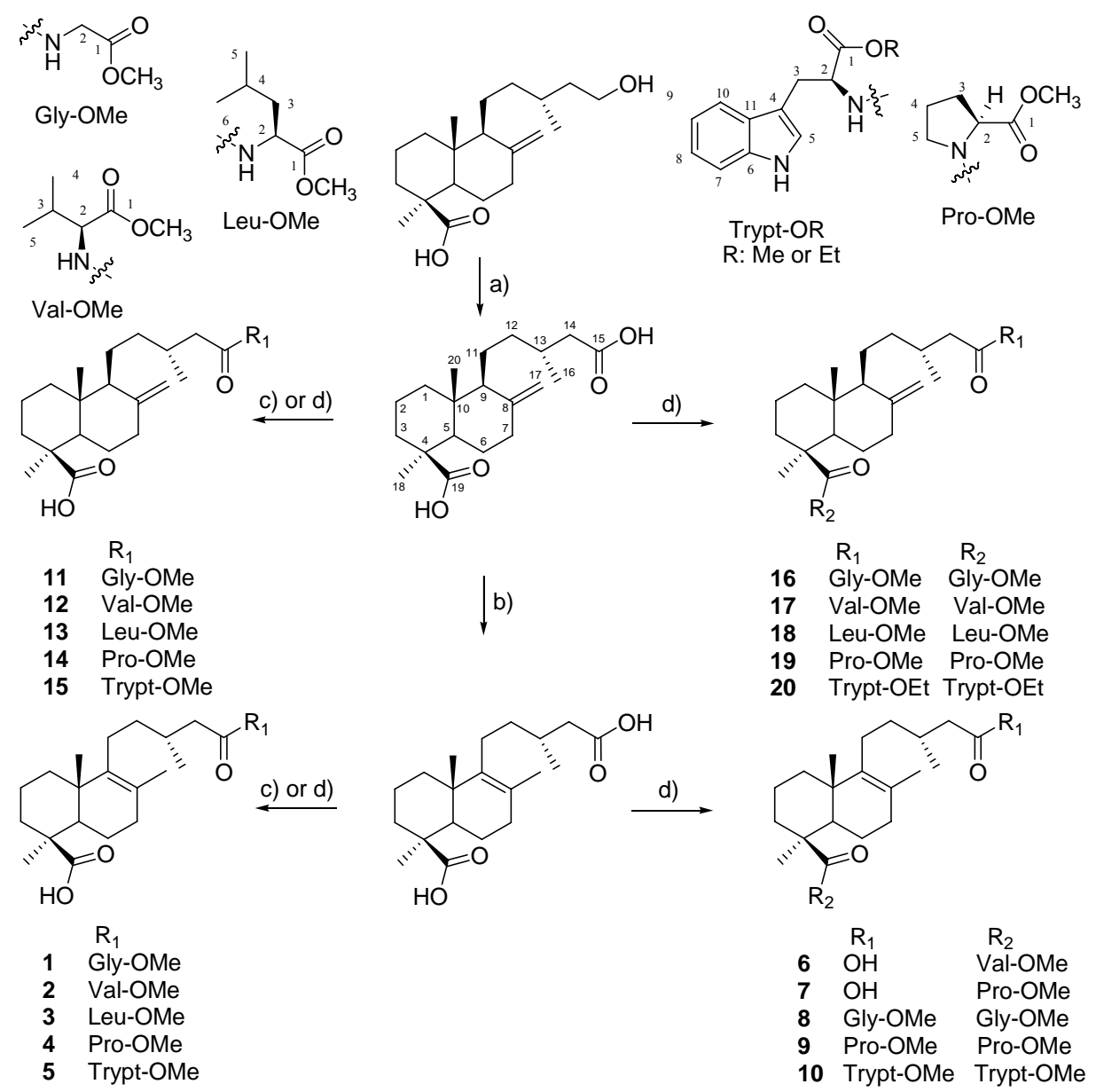

Reagents and conditions: (a) Jones oxidation: $\mathrm{CrO}_{3}, \mathrm{H}_{2} \mathrm{SO}_{4}, \mathrm{H}_{2} \mathrm{O}$, acetone; (b) $\mathrm{CH}_{3} \mathrm{COOH} / \mathrm{HBr}$, rt, $24 \mathrm{~h}$; (c) appropriate amino acid ester, DCC, DMAP, $\mathrm{CH}_{2} \mathrm{Cl}_{2}$; (d) (i) $(\mathrm{COCl})_{2}, \mathrm{CH}_{2} \mathrm{Cl}_{2}, 24 \mathrm{~h}$; (ii) appropriate amino acid ester, $\mathrm{Et}_{3} \mathrm{~N}, \mathrm{CH}_{2} \mathrm{Cl}_{2}, 48 \mathrm{~h}$. Abbreviations: Gly-OMe: Glycine methyl ester; Val-OMe: Valine methyl ester; Leu-OMe: Leucine methyl ester; Pro-OMe: Proline methyl ester; Trypt-OMe: Tryptophan methyl ester (compounds 5, 10 and 15); Trypt-OEt: Tryptophan ethyl ester (compound 20). 


\section{Results and Discussion}

Starting from junicedric acid and its 8(9)-en isomer, 20 new diterpene amides were synthesized from amino acids esters and 18 from them were investigated for gastroprotective effects and cytotoxicity (Figure 2).

To select the dose for comparing the gastroprotective effect of the new compounds, junicedric acid C-15 leucyl methyl ester amide (compound 13) and C-15,19 dileucyl methyl ester amide (compound 18) (amino acid $\mathrm{COOH}$ as methyl ester) were assessed as gastroprotective agents at three oral doses: 25,50 and $100 \mathrm{mg} / \mathrm{kg}$. In the dose-response study, the higher doses of both compounds reduced gastric lesions by $79-89 \%$ and did not differ statistically from each other. At $25 \mathrm{mg} / \mathrm{kg}$, compound 13 reduced gastric lesions by $65.6 \%$ (lesion index in $\mathrm{mm}: 15.8 \pm 6.2$ ) and compound 18 by $49.6 \%$ (lesion index in $\mathrm{mm}: 23.2 \pm 12.9$ ). Under the same experimental conditions, the lesion index of untreated animals (in $\mathrm{mm}$ ) was $46.0 \pm 5.7$ and the standard compound lansoprazole at $20 \mathrm{mg} / \mathrm{kg}$ reduced lesions by $79.3 \%$ (lesion index: $9.5 \pm 2.5$ ). Based on the results, the efficacy of the compounds preventing gastric lesions in mice was compared at the single oral dose of $25 \mathrm{mg} / \mathrm{kg}$ (Table 1).

Table 1. Gastroprotective effect of the compounds 1-5, 7-16, 18-20 at $25 \mathrm{mg} / \mathrm{kg}$ on the $\mathrm{HCl} / \mathrm{EtOH}$ induced gastric lesion model in mice.

\begin{tabular}{lcc}
\hline \multirow{2}{*}{ Compound } & \multicolumn{2}{c}{ Gastroprotective effect $^{\mathbf{a}}$} \\
\cline { 2 - 3 } & Lesion index & \% Lesion reduction $^{\text {1 }}$ \\
$\mathbf{1}$ & $12.3 \pm 7.6^{* *}$ & 73.3 \\
$\mathbf{2}$ & $19.1 \pm 8.3^{*}$ & 58.5 \\
$\mathbf{3}$ & $12.6 \pm 6.8^{* *}$ & 72.6 \\
$\mathbf{4}$ & $13.4 \pm 6.8^{* *}$ & 70.9 \\
$\mathbf{5}$ & $34.3 \pm 12.0$ & 25.4 \\
$\mathbf{7}$ & $21.2 \pm 9.5^{*}$ & 53.9 \\
$\mathbf{8}$ & $17.5 \pm 9.9^{* *}$ & 62.0 \\
$\mathbf{9}$ & $18.4 \pm 11.3^{* *}$ & 60.0 \\
$\mathbf{1 0}$ & $17.6 \pm 7.8^{* *}$ & 61.7 \\
$\mathbf{1 1}$ & $12.9 \pm 8.1^{* *}$ & 71.9 \\
$\mathbf{1 2}$ & $15.4 \pm 8.8^{* *}$ & 66.5 \\
$\mathbf{1 3}$ & $15.8 \pm 6.2^{* *}$ & 65.6 \\
$\mathbf{1 4}$ & $18.5 \pm 11.8^{*}$ & 59.8 \\
$\mathbf{1 5}$ & $12.1 \pm 4.7^{* *}$ & 73.7 \\
$\mathbf{1 6}$ & $12.2 \pm 6.9^{* *}$ & 73.5 \\
$\mathbf{1 8}$ & $23.2 \pm 12.9^{*}$ & 49.6 \\
$\mathbf{1 9}$ & $5.6 \pm 3.2^{* *}$ & 87.8 \\
$\mathbf{2 0}$ & $14.3 \pm 10.7^{*}$ & 68.9 \\
Control & $46.0 \pm 5.7$ & 0 \\
Lansoprazole & $9.5 \pm 2.5^{* *}$ & 79.3 \\
\hline
\end{tabular}

${ }^{a}$ Gastroprotective effect shown as mean lesion index \pm standard deviation (SD) and percent lesion reduction compared with untreated controls. ${ }^{b}$ Reference drug: lansoprazole at $20 \mathrm{mg} / \mathrm{kg}$. Significance set at $* \mathrm{P}<0.05 ; * * \mathrm{P}<0.01$.

For the junicedric acid 8(9)-en isomers, when the $\mathrm{COOH}$ function at $\mathrm{C}-19$ was free, a higher gastroprotective activity was observed for the glycyl (1, 73.3\%), leucyl (3, 72.6\%) and prolyl ester (4, $70.9 \%$ ) amides, which were more effective as gastroprotective agents than the corresponding C-15,19diamides. However, for the tryptophanyl ester derivatives, better gastroprotection was found for the 
diamide 10 (61.7\%) than for the monoamide 5 (25.4\%), with lower cytotoxicity seen for compound 10. For the diamides with glycine, proline and tryptophan ester, better gastroprotection was observed for the derivatives with the exo methylene group.

Under our experimental conditions and at a single oral dose of $25 \mathrm{mg} / \mathrm{kg}$, the best gastroprotective effect measured as percent lesion reduction was observed for compounds 1 (73.3\%), 3 (72.6\%), 4 (70.9\%), 11 (71.9\%), 15 (73.7\%), 16 (73.5\%) and 19 (87.8\%). Compounds 12 (66.5\%), 13 (65.6\%), and $20(68.9 \%)$ also presented good activity. Among the amide derivatives of junicedric acid with a free $\mathrm{COOH}$ function at $\mathrm{C}-19$ (compounds 11-15), higher lesion reduction was observed for compounds 11 (71.9\%), 15 (73.7\%) and 16 (73.5\%) while for the C-15,19 diamides the proline ester derivative 19 (87.8\%) and the tryptophan ester derivative 20 (68.9\%) showed better gastroprotective effects.

When considering cytotoxicity (Table 2), significant differences can be observed between the compounds. Lower cytotoxicity with $\mathrm{IC}_{50}$ values $>1,000 \mu \mathrm{M}$ towards all cell lines was observed for the tryptophan ester monoamide $\mathbf{1 5}$ and the diamides $\mathbf{1 0}$ and 20, making these derivatives the most interesting compounds when looking for gastroprotective products with low cell toxicity.

Table 2. Cytotoxicity $\left(\mathrm{IC}_{50}\right.$ values, $\left.\mu \mathrm{M}\right)$ of the compounds 1-5, 7-16, 18-20.

\begin{tabular}{lccc}
\hline \multirow{2}{*}{ ompound } & \multicolumn{3}{c}{ Cytotoxicity $\mathbf{I C}_{\mathbf{5 0}}(\boldsymbol{\mu M})^{\mathbf{b}}$} \\
\cline { 2 - 4 } & MRC-5 & AGS & Hep G2 \\
\hline $\mathbf{1}$ & $447 \pm 26$ & $189 \pm 9$ & $242 \pm 13$ \\
$\mathbf{2}$ & $>1,000$ & $>1,000$ & $>1,000$ \\
$\mathbf{3}$ & $>1,000$ & $445 \pm 27$ & $806 \pm 32$ \\
$\mathbf{4}$ & $288 \pm 11$ & $167 \pm 7$ & $321 \pm 14$ \\
$\mathbf{5}$ & $260 \pm 15$ & $149 \pm 7$ & $291 \pm 11$ \\
$\mathbf{7}$ & $459 \pm 32$ & $266 \pm 16$ & $364 \pm 15$ \\
$\mathbf{8}$ & $890 \pm 45$ & $592 \pm 30$ & $841 \pm 42$ \\
$\mathbf{9}$ & $119 \pm 7$ & $82 \pm 4$ & $67 \pm 3$ \\
$\mathbf{1 0}$ & $>1,000$ & $>1,000$ & $>1,000$ \\
$\mathbf{1 1}$ & $>1,000$ & $361 \pm 18$ & $541 \pm 22$ \\
$\mathbf{1 2}$ & $380 \pm 19$ & $309 \pm 18$ & $673 \pm 34$ \\
$\mathbf{1 3}$ & $449 \pm 29$ & $308 \pm 17$ & $808 \pm 48$ \\
$\mathbf{1 4}$ & $>1,000$ & $297 \pm 14$ & $894 \pm 56$ \\
$\mathbf{1 5}$ & $>1,000$ & $>1,000$ & $>1,000$ \\
$\mathbf{1 6}$ & $>1,000$ & $483 \pm 29$ & $529 \pm 36$ \\
$\mathbf{1 8}$ & $495 \pm 35$ & $>1000$ & $>1000$ \\
$\mathbf{1 9}$ & $267 \pm 14$ & $206 \pm 10$ & $316 \pm 16$ \\
$\mathbf{2 0}$ & $>1,000$ & $>1,000$ & $>1,000$ \\
Lansoprazole & $306 \pm 11$ & $162 \pm 6$ & $221 \pm 9$ \\
\hline
\end{tabular}

${ }^{a}$ Cultured human cell lines: MRC-5, human normal lung fibroblasts; AGS, gastric epithelial adenocarcinoma cells; Hep G2, hepatocytes. ${ }^{\mathrm{b}}$ Values are arithmetic means of three different experiments in quadruplicate $\pm \mathrm{SD}$. Confluent cultures were treated with the culture medium containing the compounds at concentrations ranging between 0 and $1000 \mu \mathrm{M}$ for $24 \mathrm{~h}$. Cell viability was determined by the neutral red uptake assay. ${ }^{c}$ Lansoprazole was used as the reference drug. 
Compound 9 proved to be the more cytotoxic derivative, with $\mathrm{IC}_{50}$ values of 119,82 and $67 \mu \mathrm{M}$ against human fibroblasts, AGS cells and Hep G2 hepatocytes, respectively. None of the new compounds presented selectivity against the cell panel used, but higher gastroprotective effect with lower toxicity was found for compounds 15 and 20. Compound 19, the most active product of this study, presented a cytotoxicity comparable with that of the reference compound lansoprazole, but higher than that of compounds 15 and 20, which presented $\mathrm{IC}_{50}$ values $>1,000 \mu \mathrm{M}$.

\section{Experimental}

\subsection{General}

Optical rotations were obtained for solutions in $\mathrm{CHCl}_{3}$ (concentrations expressed in $\mathrm{g} / 100 \mathrm{~mL}$ ) on a Jasco DIP 370 polarimeter. Infrared spectra were recorded on a Nicolet Nexus FT-IR instrument (Thermo Electron Corporation). NMR spectra were recorded at room temperature in $\mathrm{CDCl}_{3}$ using a Bruker Avance 400 instrument (Bruker, Germany) operating at $400 \mathrm{MHz}$ for ${ }^{1} \mathrm{H}$ and $100 \mathrm{MHz}$ for ${ }^{13} \mathrm{C}$ spectra. All chemical shifts values are reported relative to residual $\mathrm{CHCl}_{3}$.Chemical shifts $(\delta)$ are given in ppm and coupling constants $(\mathrm{J})$ are reported in Hertz. Mass spectra were measured with an EBE trisector VG Autospec Micromass spectrometer operating at $70 \mathrm{eV}$ and are presented as $\mathrm{m} / \mathrm{z}$ (rel. int. \%). Silica gel 60 (Merck, 63-200 $\mu \mathrm{m}$ particle size) was used for column chromatography, precoated silica gel plates (Merck, Kieselgel $60 \mathrm{~F}_{254}, 0.25 \mathrm{~mm}$ ) were used for thin layer chromatography (TLC) analysis. Reversed-phase silica gel $100 \mathrm{C}_{8}$ (Fluka, Germany) was used to purify the the amides. TLC spots were visualized by spraying the chromatograms with p-anisaldehyde-ethanol-acetic acid- $\mathrm{H}_{2} \mathrm{SO}_{4}$ $(2: 170: 20: 10 \mathrm{v} / \mathrm{v})$ and heating at $110^{\circ} \mathrm{C}$ for $3 \mathrm{~min}$.

\subsection{Synthesis of the diterpene amides 1-20}

The diterpene used as starting compound for the synthesis was obtained from the resin of Araucaria araucana as described in previous work [14,15]. 15-Hydroxyimbricatolic acid was treated with Jones reagent $\left(\mathrm{CrO}_{3} / \mathrm{H}_{2} \mathrm{SO}_{4} / \mathrm{H}_{2} \mathrm{O}\right)$ to afford after purification by column chromatography on silica gel the diacid diterpene junicedric acid. The 8(9)-en junicedric acid isomer was prepared treating junicedric acid with $\mathrm{HBr}$ in acetic acid. The amides described herein for the first time were prepared under an inert $\left(\mathrm{N}_{2}\right)$ atmosphere. Briefly, the diterpene was dissolved in dry dichloromethane $\left(\mathrm{CH}_{2} \mathrm{Cl}_{2}, \mathrm{DCM}\right)$ and ice cooled under nitrogen flow. To this solution, oxalyl chloride in dry DCM was added dropwise with stirring. The volume of dry DCM used to dissolve the diterpene was about 15-20 mL. Oxalyl chloride was dissolved in dry DCM in a 1:5 ratio The diterpene:oxalyl chloride molar ratio was 1:40. The mixture was stirred at room temperature overnight, then the DCM was evaporated and the residue vacuum dried. The dry residue was dissolved in dry DCM and the $C$-protected amino acid ester (AAE) (as hydrochloride) was added as well as triethylamine (TEA) under constant $\mathrm{N}_{2}$ flow. The diterpene:AAE:TEA molar ratio was 1:3:3. The reaction mixture was left at room temperature $\left(18-20^{\circ} \mathrm{C}\right)$ under stirring and inert atmosphere for two days $(48 \mathrm{~h})$. Then, the mixture was washed two times with water, and the aqueous phase extracted with DCM to obtain the crude reaction mixture $[21,22]$. To prepare the C-15 monoamides, the diterpene dissolved in dry DCM was treated with $N, N^{\prime}-$ dicyclohexylcarbodiimide (DCC)/dimethylaminopyridine (DMAP) and the amino acid ester.HCl. The 
reaction time was 1-3 $\mathrm{h}$ and the process was monitored by TLC. Purification was undertaken using a combination of gel permeation in Sephadex LH-20, eluting with an petroleum ether (PE):DCM:MeOH 1:1:1 mixture, silica gel column chromatography (CC) and reversed-phase silica gel chromatography (RP-8) to afford the amides. The reagents and conditions used are summarized in Figure 2. The structure of the compounds was determined mainly by NMR spectroscopy and by comparing the spectroscopic data with those of similar compounds already reported and by IR and mass spectra.

Labd-8(9)-en-15,19-dioic acid, 15-glycyl methyl ester amide (1). The diterpene diacid (0.288 g, 0.857 mmol) was treated with oxalyl chloride to yield the acid chloride who was treated with glycine methyl ester.HCl)/TEA to afford after $24 \mathrm{~h}$ compound 1 (0.266 g, $0.653 \mathrm{mmol}, 76.2 \%$ yield, Rf 0.08 , PE:EtOAc 6:4) as a colourless resin. Using DCC/DMAP, we did not obtain the desired product. $[\alpha]_{D}^{20}$ : +45.5 (c 0.176, $\mathrm{CHCl}_{3}$ ); IR $v_{\max }$ (film) 3,311, 2,952, 2,872, 1,792, 1,748, 1,653, 1,537, 1,433, 1,202, 991, $748 \mathrm{~cm}^{-1} ;{ }^{1} \mathrm{H}-\mathrm{NMR}\left(\mathrm{CDCl}_{3}\right) \delta 0.94(3 \mathrm{H}, \mathrm{s}, \mathrm{H}-20), 1.01(3 \mathrm{H}, \mathrm{d}, J=6.4 \mathrm{~Hz}, \mathrm{H}-16), 1.32$ (3H, s, H18), 1.59 (3H, s, H-17), 3.80 (3H, s), 4.05 (1H, dd, $J=18.8,5.2 \mathrm{~Hz}$, Gly H-2), 4.10 (1H, dd, $J=18.8$, $5.2 \mathrm{~Hz}$, Gly H-2), $5.98(1 \mathrm{H}$, dd $J=4.8,4.8 \mathrm{~Hz}, \mathrm{NH}) ;{ }^{13} \mathrm{C}-\mathrm{NMR}\left(\mathrm{CDCl}_{3}\right): 37.63$ (t, C-1), 19.64 (t, C-2), 38.09 (t, C-3), 45.64 (s, C-4), 53.99 (d, C-5), 21.14 (t, C-6), 37.34 (t, C-7), 126.63 (s, C-8), 139.56 (s, C-9), 39.87 (s, C-10), 25.90 (t, C-11), 34.52 (t, C-12), 31.99 (d, C-13), 44.17 (t, C-14), 173.55 (s, C15), 19.87 (q, C-16), 19.64 (q, C-17), 27.99 (q, C-18), 170.78 (s, C-19), 19.06 (q, C-20); Gly: 172.84 (s, C-1'), 41.40 (t, C-2'); 52.57 (q, OMe); ESI-MS (m/z): 430.0926. Calcd for $\left[\mathrm{C}_{23} \mathrm{H}_{37} \mathrm{NO}_{5} \mathrm{Na}^{+}\right]$: 430.2570 .

Labd-8(9)-en-15,19-dioic acid, 15-valyl methyl ester amide (2). Some $0.457 \mathrm{~g}$ of the diterpene diacid (1.36 mmol) afforded after treatment with DCC/DMAP and valine methyl ester.HCl, the compound 2 (0.242 g, 39\% yield, Rf 0.33, PE:EtOAc 6:4) as a colourless resin. $[\alpha]_{D}^{20}:+52.8\left(c 0.843, \mathrm{CHCl}_{3}\right)$; IR $v_{\max }$ (film) 3,334, 2,956, 2,932, 2,872, 1,796, 1,740, 1,649, 1,537, 1,465, 1,437, 1,382, 1,206, 995, 756 $\mathrm{cm}^{-1}$; ${ }^{1} \mathrm{H}-\mathrm{NMR}\left(\mathrm{CDCl}_{3}\right) \delta 0.88(3 \mathrm{H}, \mathrm{s}, \mathrm{H}-20), 0.91(6 \mathrm{H}, \mathrm{d}, J=6.8 \mathrm{~Hz}, \mathrm{Val} \mathrm{H}-4, \mathrm{H}-5), 0.95(3 \mathrm{H}, \mathrm{d}$, $J=6.4 \mathrm{~Hz}, \mathrm{H}-16), 1.27$ (3H, s, H-18), 1.53 (3H, s, H-17), 2.16-2.18 (1H, m, Val H-3), 3.71 (3H, s), $4.56\left(1 \mathrm{H}, \mathrm{dd}, J=8.8,4.8 \mathrm{~Hz}\right.$, Val H-2), $5.87(1 \mathrm{H}, \mathrm{d}, J=8.8 \mathrm{~Hz}, \mathrm{NH}) ;{ }^{13} \mathrm{C}-\mathrm{NMR}\left(\mathrm{CDCl}_{3}\right): 37.50(\mathrm{t}, \mathrm{C}-$ 1), 19.40 (t, C-2), 37.90 (t, C-3), 45.44 (s, C-4), 53.82 (d, C-5), 20.95 (t, C-6), 37.16 (t,C-7), 126.39 (s, C-8), 139.37 (s, C-9), 39.69 (s, C-10), 25.69 (t, C-11), 34.33 (t, C-12), 31.86 (d, C-13), 44.37 (t, C-14), 172.74 (s, C-15), 19.48 (q, C-16), 19.70 (q, C-17), 27.81 (q, C-18), 173.34 (s, C-19), 18.96 (q, C-20); Val: 172.37 (s, C-1'), 56.88 (d, C-2'), 31.33 (d, C-3'), 18.88 (q, C-4'), 17.90 (q, C-5'); 52.12 (q, $\mathrm{OMe})$; $\operatorname{ESI}-\mathrm{MS}(\mathrm{m} / \mathrm{z})$ : 472.6656. Calcd for $\left[\mathrm{C}_{26} \mathrm{H}_{43} \mathrm{NO}_{5} \mathrm{Na}^{+}\right]: 472.3039$.

Labd-8(9)-en-15,19-dioic acid, 15-leucyl methyl ester amide (3). Treatment of the diacid (0.401 g, $1.19 \mathrm{mmol})$ with DCC/DMAP and leucine methyl ester.HCl afforded 3 (0.313 g, $0.67 \mathrm{mmol}, 56.7 \%$ yield, Rf 0.28, PE:EtOAc 6:4) as a colourless resin. $[\alpha]_{D}^{20}:+55.33$ (c 0.459, $\mathrm{CHCl}_{3}$ ); IR $v_{\max }$ (film) 3,307, 2,952, 2,932, 2,868, 1,796, 1,744, 1,649, 1,537, 1,378, 995, $756 \mathrm{~cm}^{-1} ;{ }^{1} \mathrm{H}-\mathrm{NMR}\left(\mathrm{CDCl}_{3}\right) \delta 0.94$ (3H, s, H-20), 0.96 (3H, d, $J=6.0 \mathrm{~Hz}$, Leu H-6), 0.97 (3H, d, $J=6.0 \mathrm{~Hz}$, Leu H-5), 1.00 (3H, d, $J=5.6 \mathrm{~Hz}, \mathrm{H}-16), 1.32(3 \mathrm{H}, \mathrm{s}, \mathrm{H}-18), 1.59$ (3H, s, H-17), $3.76(3 \mathrm{H}, \mathrm{s}), 4.69$ (1H, ddd, $J=5.2,8.6,8.8$ $\mathrm{Hz}$, Leu H-2), $5.83(1 \mathrm{H}$, br d, $J=5.6 \mathrm{~Hz}, \mathrm{NH}) ;{ }^{13} \mathrm{C}-\mathrm{NMR}\left(\mathrm{CDCl}_{3}\right): 37.66$ (t, C-1), 19.60 (t, C-2), 38.07 (t, C-3), 45.60 (s, C-4), 53.99 (d, C-5), 21.11 (t, C-6), 37.34 (t, C-7), 126.52 (s, C-8), 139.58 (s, C-9), 
39.84 (s, C-10), 26.19 (t, C-11), 34.50 (t, C-12), 32.00 (d, C-13), 44.40 (t, C-14), 172.42 (s, C-15), 23.00 (q, C-16), 19.83 (q, C-17), 27.97 (q, C-18), 173.88 (s, C-19), 19.04 (q, C-20); Leu: 173.47 (s, C1'), 50.72 (d, C-2'), 41.98 (t, C-3'), 22.11 (d, C-4'), 19.61 (q, C-5'), 19.61 (q, C-6'); 52.38 (q, OMe); ESI-MS (m/z): 486.3339. Calcd for $\left[\mathrm{C}_{27} \mathrm{H}_{45} \mathrm{NO}_{5} \mathrm{Na}^{+}\right]$: 486.3195 .

Labd-8(9)-en-15,19-dioic acid, 15-prolyl methyl ester amide (4). Treatment of the diacid (331 mg, $0.985 \mathrm{mmol})$ with DCC/DMAP and proline methyl ester. $\mathrm{HCl}$ yielded compound 4 (55.2 $\mathrm{mg}, 0.123$ mmol, $12.5 \%$ yield, Rf 0.10, PE:EtOAc 6:4) as colourless resins. $[\alpha]_{D}^{20}:+34.24\left(c 0.552, \mathrm{CHCl}_{3}\right)$; IR $v_{\max }$ (film) 3,454, 2,948, 2,872, 1,796, 1,748, 1,641, 1,429, 1,413, 1,374, 1,198, 1,178, 991, $748 \mathrm{~cm}^{-1}$; ${ }^{1} \mathrm{H}-\mathrm{NMR}\left(\mathrm{CDCl}_{3}\right) \delta 0.93(3 \mathrm{H}, \mathrm{s}, \mathrm{H}-20), 1.01(3 \mathrm{H}, \mathrm{d}, J=6.4 \mathrm{~Hz}, \mathrm{H}-16), 1.31$ (3H, s, H-18), 1.59 (3H, s, H-17), 3.53 (1H, m, Pro H-5), 3.67 (1H, m, Pro H-5), 3.74 (3H, s), 4.50 (1H, dd, $J=8.0,3.2$ Hz, Pro $\mathrm{H}-2) ;{ }^{13} \mathrm{C}-\mathrm{NMR}\left(\mathrm{CDCl}_{3}\right): 37.76$ (t, C-1), 19.55 (t, C-2), 38.06 (t, C-3), 45.59 (s, C-4), 53.98 (d, C-5), 21.11 (t, C-6), 34.49 (t, C-7), 126.46 (s, C-8), 139.65 (s, C-9), 39.83 (s, C-10), 25.80 (t, C-11), 37.31 (t, C-12), 31.43 (d, C-13), 41.73 (t, C-14), 171.71 (s, C-15), 19.71 (q, C-16), 19.85 (q, C-17), 27.94 (q, C-18), 173.49 (s, C-19), 19.04 (q, C-20); Pro: 173.10 (s, C-1'); 58.78 (d, C-2'); 29.37 (t, C-3'); 24.98 (t, C-4'); 47.39 (t, C-5'); 52.24 (q, OMe); ESI-MS (m/z): 470.1352. Calcd for $\left[\mathrm{C}_{26} \mathrm{H}_{41} \mathrm{NO}_{5} \mathrm{Na}^{+}\right]$: 470.2882 .

Labd-8(9)-en-15,19-dioic acid, 15-tryptophanyl methyl ester amide (5). Some $483 \mathrm{mg}$ of the diacid (1.44 mmol) was treated with DCC/DMAP and tryptophane methyl ester.HCl to afford 5 as a colourless resin $\left(62 \mathrm{mg}, 0.116 \mathrm{mmol}, 8 \%\right.$ yield, Rf 0.21, PE:EtOAc 6:4). $[\alpha]_{D}^{20}:+0.81(c 0.618$, $\mathrm{CHCl}_{3}$ ); IR $v_{\max }$ (film) 3,314, 2,928, 2,852, 1,736, 1,692, 1,645, 1,557, 1,517, 1,453, 1,437, 1,378, 1,218, $756 \mathrm{~cm}^{-1} ;{ }^{1} \mathrm{H}-\mathrm{NMR}\left(\mathrm{CDCl}_{3}\right) \delta 0.82(3 \mathrm{H}, \mathrm{s}, \mathrm{H}-20), 0.86(3 \mathrm{H}, \mathrm{d}, J=6.0 \mathrm{~Hz}, \mathrm{H}-16), 1.24(3 \mathrm{H}, \mathrm{s}$, H-18), 1.51 (3H, s, H-17), 3.28 (2H, m, Tript H-3), $3.66(3 \mathrm{H}, \mathrm{s}), 4.96(1 \mathrm{H}, \mathrm{m}$, Tript H-2), 5.99 (1H, br $\mathrm{d}, J=7.2 \mathrm{~Hz}$, Tript NH), $6.95(1 \mathrm{H}$, br s, Tript H-5), $7.08(1 \mathrm{H}, \mathrm{dd}, J=7.8,7.1 \mathrm{~Hz}$, Tript H-8), 7.16 $(1 \mathrm{H}, \mathrm{dd}, J=7.5,7.2 \mathrm{~Hz}$, Tript H-9), $7.32(1 \mathrm{H}, \mathrm{d}, J=8.1 \mathrm{~Hz}$, Tript H-7), $7.50(1 \mathrm{H}, \mathrm{d}, J=8.2 \mathrm{~Hz}$, Tript $\mathrm{H}-10), 8.28$ (1H, br s, NH); ${ }^{13} \mathrm{C}-\mathrm{NMR}\left(\mathrm{CDCl}_{3}\right): 37.67$ (t, C-1), 19.72 (t, C-2), 37.75 (t, C-3), 43.94 (s, C-4), 53.79 (d, C-5), 20.95 (t, C-6), 37.37 (t, C-7), 126.77 (s, C-8), 139.39 (s, C-9), 39.96 (s, C-10), 25.03 (t, C-11), 34.45 (t, C-12), 31.94 (d, C-13), 44.24 (t, C-14), 172.64 (s, C-15), 19.56 (q, C-16), 19.91 (q, C-17), 28.82 (q, C-18), 183.68 (s, C-19), 18.14 (q, C-20); Trypt: 172.80 (s, C-1'); 53.06 (d, C-2'); 27.91 (t, C-3'); 110.25 (s, C-4'); 122.91 (d, C-5'); 136.34 (s, C-6'); 111.51 (d, C-7'); 119.86 (d, C-8'); 122.43 (d, C-9'); 118.73 (d, C-10'); 127.90 (s, C-11'); 52.53 (q, OMe); ESI-MS (m/z): 559.3320. Calcd for $\left[\mathrm{C}_{32} \mathrm{H}_{44} \mathrm{~N}_{2} \mathrm{O}_{5} \mathrm{Na}^{+}\right]$: 559.3250 .

Labd-8(9)-en-15,19-dioic acid, 19-valyl methyl ester amide (6). The diacid (210 mg. $0.61 \mathrm{mmol})$ was treated with oxalyl chloride to afford the acid chloride. After reaction with valine methyl ester.HCl/TEA and purification, the compound 6 was obtained as a colourless resin (57 $\mathrm{mg}$, 0.127 mmol, 21\% yield, Rf: 0.23 (PE:EtOAc 7:3). $[\alpha]_{D}^{20}:+70\left(c 0.057, \mathrm{CHCl}_{3}\right.$ ); IR $v_{\max }$ (film) 3318, 2960, 2932, 2880, 1740, 1724, 1692, 1641, 1537, 1210, 1154, $748 \mathrm{~cm}^{-1} ;{ }^{1} \mathrm{H}$ NMR $\left(\mathrm{CDCl}_{3}\right) \delta 0.80(3 \mathrm{H}$, s, H-20), 0.87 (6H, d, $J=6.9 \mathrm{~Hz}$, Val H-4, H-5), 0.91 (3H, d, $J=6.4$ Hz, H-16), 1.19 (3H, s, H-18), 1.50 (3H, s, H-17), 3.69 (3H, s), $4.56(1 \mathrm{H}, \mathrm{dd}, J=8.8,5.0 \mathrm{~Hz}, \mathrm{Val} \mathrm{H}-2), 6.14(1 \mathrm{H}, \mathrm{d}, J=8.8 \mathrm{~Hz}, \mathrm{NH})$; ${ }^{13} \mathrm{C} \mathrm{NMR}\left(\mathrm{CDCl}_{3}\right): 37.63$ (t, C-1), 19.69 (t, C-2), 37.67 (t, C-3), 43.91 (s, C-4), 53.71 (d, C-5), 20.91 
(t, C-6), 37.36 (t, C-7), 126.71 (s, C-8), 139.36 (s, C-9), 39.89 (s, C-10), 25.83 (t, C-11), 34.41 (t, C12), 32.01 (d, C-13), 44.37 (t, C-14), 173.12 (s, C-15), 19.59 (q, C-16), 19.88 (q, C-17), 28.82 (q, C18), 183.64 (s, C-19), 19.09 (q, C-20); Val: 172.89 (s, C-1'), 57.04 (d, C-2'), 31.45 (d, C-3'), 18.14 (q, C-4'), 18.05 (q, C-5'); 52.31 (q, OMe); ESI-MS (m/z): 472.3318. Calcd for $\left[\mathrm{C}_{26} \mathrm{H}_{43} \mathrm{NO}_{5} \mathrm{Na}^{+}\right]$: 472.3039 .

Labd-8(9)-en-15,19-dioic acid, 19-prolyl methyl ester amide (7). For preparation please see under compound 9. Colorless resin; $[\alpha]_{D}^{20}:+26.0$ (c 1.02, $\mathrm{CHCl}_{3}$ ); IR $v_{\max }$ (film) 3,420, 2,952, 2,876, 1,792, 1,740, 1,641, 1,437, 1,198, 1,174, 991, $756 \mathrm{~cm}^{-1} ;{ }^{1} \mathrm{H}-\mathrm{NMR}\left(\mathrm{CDCl}_{3}\right) \delta 0.81(3 \mathrm{H}, \mathrm{s}, \mathrm{H}-20), 0.95(3 \mathrm{H}, \mathrm{d}$, $J=6.4 \mathrm{~Hz}, \mathrm{H}-16), 1.20$ (3H, s, H-18), 1.52 (3H, s, H-17), 3.49 (1H, m, Pro H-5), 3.62 (1H, m, Pro H5), $3.67(3 \mathrm{H}, \mathrm{s}), 4.46\left(1 \mathrm{H}, \mathrm{dd}, J=8.4,3.6 \mathrm{~Hz}\right.$, Pro H-2); ${ }^{13} \mathrm{C}-\mathrm{NMR}\left(\mathrm{CDCl}_{3}\right): 37.48$ (t, C-1), 19.51 (t, C-2), 37.68 (t, C-3), 43.77 (s, C-4), 53.49 (d, C-5), 20.76 (t, C-6), 34.27 (t, C-7), 126.51 (s, C-8), 139.34 (s, C-9), 39.75 (s, C-10), 25.70 (t, C-11), 37.20 (t, C-12), 31.36 (d, C-13), 41.57 (t, C-14), 171.84 (s, C-15), 19.51 (q, C-16), 19.74 (q, C-17), 28.68 (q, C-18), 183.64 (s, C-19), 17.98 (q, C-20); Pro: 172.96 (s, C-1'); 58.64 (d, C-2'); 29.23 (t, C-3'); 24.81 (t, C-4'); 47.29 (t, C-5'); 52.14 (q, $\mathrm{OMe})$; ESI-MS (m/z): 470.1352. Calcd for $\left[\mathrm{C}_{26} \mathrm{H}_{41} \mathrm{NO}_{5} \mathrm{Na}^{+}\right]$: 470.2882.

Labd-8(9)-en-15,19-dioic acid, 15,19-diglycyl methyl ester amide (8). The diacid (203 mg, $0.604 \mathrm{mmol}$ ) was treated with oxalyl chloride to afford the acid chloride. After reaction with glycine methyl ester.HCl/TEA and purification on Sephadex LH-20, the compound 8 was obtained as a colourless resin. (68 mg, $0.142 \mathrm{mmol}, 24 \%$ yield, Rf: 0.20 (PE:EtOAc 1:1). $[\alpha]_{D}^{20}:+69(c 0.056$, $\mathrm{CHCl}_{3}$ ); IR $v_{\text {max }}$ (film) 3,382, 3,334, 2,944, 2,860, 1,752, 1,645, 1,521, 1,437, 1,366, 1,206, $752 \mathrm{~cm}^{-1}$; ${ }^{1} \mathrm{H}-\mathrm{NMR}\left(\mathrm{CDCl}_{3}\right) \delta 0.80(3 \mathrm{H}, \mathrm{s}, \mathrm{H}-20), 0.94(3 \mathrm{H}, \mathrm{d}, J=6.3 \mathrm{~Hz}, \mathrm{H}-16), 1.18(3 \mathrm{H}, \mathrm{s}, \mathrm{H}-18), 1.53(3 \mathrm{H}, \mathrm{s}$, H-17), 3.74 (6H, s), 3.97 (2H, m, Gly H-2), 4.05 (2H, m, Gly H-2), 6.11 (1H, t, $J=4.8 \mathrm{~Hz}, \mathrm{NH}), 6.16$ $(1 \mathrm{H}, \mathrm{t}, J=4.8 \mathrm{~Hz}, \mathrm{NH}) ;{ }^{13} \mathrm{C}-\mathrm{NMR}\left(\mathrm{CDCl}_{3}\right): 37.70$ (t, C-1), 19.83 (t, C-2), 38.06 (t, C-3), 44.03 (s, C4), 53.90 (d, C-5), 21.41 (t, C-6), 37.59 (t, C-7), 126.40 (s, C-8), 139.61 (s, C-9), 39.92 (s, C-10), 25.84 (t, C-11), 34.65 (t, C-12), 31.94 (d, C-13), 44.03 (t, C-14), 172.88 (s, C-15), 19.56 (q, C-16), 19.85 (q, C-17), 29.76 (q, C-18), 177.38 (s, C-19), 18.17 (q, C-20); Gly: 171.03, 170.71 (s, C'-1), 41.45, 41.31 (t, C'-2); 52.36, 52.31 (q, OMe); ESI-MS (m/z): 501.3414. Calcd for $\left[\mathrm{C}_{26} \mathrm{H}_{42} \mathrm{~N}_{2} \mathrm{O}_{6} \mathrm{Na}^{+}\right]$: 501.2941.

Labd-8(9)-en-15,19-dioic acid, 15,19-diprolyl methyl ester amide (9). Treatment of the diacid (0.289 g, $0.86 \mathrm{mmol}$ ) with oxalyl chloride afforded the acid chloride which was treated with proline methyl ester.HCl /TEA to afford the monoamide 7 (90 mg, $0.2 \mathrm{mmol}, 23 \%$ yield, Rf 0.33, PE:EtOAc 1:1) and the diamide 9 (55 mg, $0.098 \mathrm{mmol}, 11.4 \%$ yield, Rf 0.53, PE:EtOAc 1:1) as colourless resins. Compound 9: $[\alpha]_{D}^{20}:+29.0\left(\mathrm{CHCl}_{3} ; \mathrm{c}=0.558\right)$; IR $v_{\max }($ film) $3370,2948,2880,1744,1720,1649$, 1433, 1194, $752 \mathrm{~cm}^{-1}$; ${ }^{1} \mathrm{H}-\mathrm{NMR}\left(\mathrm{CDCl}_{3}\right) \delta 0.76(3 \mathrm{H}, \mathrm{s}, \mathrm{H}-20), 1.00(3 \mathrm{H}, \mathrm{d}, J=6.4 \mathrm{~Hz}, \mathrm{H}-16), 1.20$ (3H, s, H-18), 1.58 (3H, s, H-17), 3.53 (1H, m, Pro H-5), 3.66 (1H, m, Pro H-5), 3.64 (3H, s), 3.73 $(3 \mathrm{H}, \mathrm{s}), 4.51\left(1 \mathrm{H}, \mathrm{dd}, J=8.8,2.8 \mathrm{~Hz}\right.$, Pro H-2); ${ }^{13} \mathrm{C}-\mathrm{NMR}\left(\mathrm{CDCl}_{3}\right): 37.84(\mathrm{t}, \mathrm{C}-1), 19.78(\mathrm{t}, \mathrm{C}-2)$, 37.94 (t, C-3), 44.07 (s, C-4), 53.79 (d, C-5), 21.05 (t, C-6), 34.50 (t, C-7), 126.66 (s, C-8), 139.56 (s, C-9), 39.73 (s, C-10), 25.85 (t, C-11), 37.42 (t, C-12), 31.48 (d, C-13), 41.77 (t, C-14), 171.78 (s, C15), 19.71 (q, C-16), 19.91 (q, C-17), 28.62 (q, C-18), 178.28 (s, C-19), 17.95 (q, C-20); Pro: 173.12 
(s, 2 C, C-1'); 58.78 (d, 2 C, C-2'); 29.38 (t, 2 C, C-3’); 24.99 (t, 2 C, C-4'); 47.40 (t, 2 C, C-5'); 52.26 (q, OMe) 51.22 (q, OMe); ESI-MS (m/z): 581.4495. Calcd for $\left[\mathrm{C}_{32} \mathrm{H}_{50} \mathrm{~N}_{2} \mathrm{O}_{6} \mathrm{Na}^{+}\right]: 581.3566$.

Labd-8(9)-en-15,19-dioic acid, 15,19-ditryptophanyl methyl ester amide (10). Some $204 \mathrm{mg}$ of the diacid $(0.607 \mathrm{mmol})$ was treated with oxalyl chloride to obtain the acid chloride and reacted with tryptophane methyl ester.HCl/TEA. After purification by $\mathrm{CC}$ on silica gel, compound $\mathbf{1 0}$ was obtained as a colourless resin $(259 \mathrm{mg}, 0.352 \mathrm{mmol}, 58 \%$ yield $) .[\alpha]_{D}^{20}:+87\left(c 0.153, \mathrm{CHCl}_{3}\right)$; IR $v_{\max }$ (film) $3414,3299,2956,2924,2868,1748,1653,1501,1461,1437,1206,752 \mathrm{~cm}^{-1} ;{ }^{1} \mathrm{H}$ NMR $\left(\mathrm{CDCl}_{3}\right) \delta$ 0.65 (3H, s, H-20), 0.85 (3H, d, $J=5.8 \mathrm{~Hz}, \mathrm{H}-16), 1.04$ (3H, s, H-18), 1.45 (3H, s, H-17), 3.27 (4 H, m, Tript H-3), $3.62(3 \mathrm{H}, \mathrm{s}), 3.64(3 \mathrm{H}, \mathrm{s}), 4.83(1 \mathrm{H}, \mathrm{m}$, Tript H-2), $4.94(1 \mathrm{H}, \mathrm{m}$, Tript H-2), $6.04(1 \mathrm{H}, \mathrm{d}$, $J=7.9 \mathrm{~Hz}$, Tript NH), $6.10(1 \mathrm{H}, \mathrm{d}, J=6.8 \mathrm{~Hz}$, Tript NH), $6.92(1 \mathrm{H}, \mathrm{d}, J=2 \mathrm{~Hz}$, Tript H-5), $6.93(1 \mathrm{H}$, $\mathrm{d}, J=2 \mathrm{~Hz}$, Tript H-5), 7.07 (2H, dd, $J=7.5,7.5 \mathrm{~Hz}$, Tript H-8), 7.15 (2H, dd, $J=7.5,7.3 \mathrm{~Hz}$, Tript H-9), $7.31(2 \mathrm{H}, \mathrm{d}, J=8.0 \mathrm{~Hz}$, Tript H-7), $7.50(1 \mathrm{H}, \mathrm{d}, J=8.2 \mathrm{~Hz}$, Tript H-10), $7.52(1 \mathrm{H}$, d, $J=8.2 \mathrm{~Hz}$, Tript H-10), $8.69(1 \mathrm{H}, \mathrm{s}, \mathrm{NH}) ;{ }^{13} \mathrm{C} \mathrm{NMR}\left(\mathrm{CDCl}_{3}\right): 37.61$ (t, C-1), 19.64 (t, C-2), 38.06 (t, C-3), 43.94 (s, C-4), 53.85 (d, C-5), 21.06 (t, C-6), 37.56 (t, C-7), 126.23 (s, C-8), 139.54 (s, C-9), 39.82 (s, C-10), 25.73 (t, C-11), 34.56 (t, C-12), 31.74 (d, C-13), 44.14 (t, C-14), 172.59 (s, C-15), 19.45 (q, C-16), 19.76 (q, C-17), 29.65 (q, C-18), 177.26 (s, C-19), 18.14 (q, C-20); Trypt: 172.97, 172.67 (s, C-1'); 53.17, 53.12 (d, C-2'); 27.84, 27.58 (t, C-3'); 109.90, 109.86 (s, C-4'); 123.02, 122.99 (d, C-5'); 136.39 (s, 2 C, C-6'); 111.59, 111.52 (d, C-7'); 119.54, 119.53 (d, C-8'); 122.26, 122.21 (d, C-9'); 118.45, 118.43 (d, C-10'); 127.78, 127.60 (s, C-11'); 52.40, 52.33 (q, OMe); ESI-MS $(\mathrm{m} / \mathrm{z}):$ 759.4151. Calcd for $\left[\mathrm{C}_{44} \mathrm{H}_{56} \mathrm{~N}_{4} \mathrm{O}_{6} \mathrm{Na}^{+}\right]: 759.4097$.

Labd-8(17)-en-15,19-dioic acid, 15-glycyl methyl ester amide (11). The diacid (1.04 mmol) was treated with oxalyl chloride to obtain the corresponding acid chloride. Treatment of the acid chloride with the glycine methyl ester.HCl /TEA yielded the amide 11 as colourless resin $(0.48 \mathrm{mmol}, 47 \%$ yield). Treatment of the diacid (215 mg, $0.64 \mathrm{mmol})$ with DCC/DMAP afforded after treatment with the AAE. $\mathrm{HCl}$ and purification the compound 11 (56 mg, $0.138 \mathrm{mmol}, 21 \%$ yield, Rf: 0.38, PE:EtOAc 1:1). $[\alpha]_{D}^{20}:+26\left(c 1.765, \mathrm{CHCl}_{3}\right)$; IR $v_{\max }$ (film) 3314, 2952, 2868, 1752, 1645, 1521, 1210, $756 \mathrm{~cm}^{-1}$; ${ }^{1} \mathrm{H}$ NMR $\left(\mathrm{CDCl}_{3}\right) \delta 0.55(3 \mathrm{H}, \mathrm{s}, \mathrm{H}-20), 0.85(3 \mathrm{H}, \mathrm{d}, J=6.0 \mathrm{~Hz}, \mathrm{H}-16), 1.17(3 \mathrm{H}, \mathrm{s}, \mathrm{H}-18), 3.66(3 \mathrm{H}$, s), $3.92(1 \mathrm{H}, \mathrm{dd}, J=18.4,4.8 \mathrm{~Hz}$, Gly H-2), 4.00 (1H, dd, $J=18.4,4.8 \mathrm{~Hz}$, Gly H-2), 4.40 (1H, s, H17), 4.74 (1H, s, H-17), $5.97(1 \mathrm{H}, \mathrm{t}, J=4.8 \mathrm{~Hz}, \mathrm{NH}) ;{ }^{13} \mathrm{C} \mathrm{NMR}\left(\mathrm{CDCl}_{3}\right): 38.85$ (t, C-1), 19.40 (t, C-2), 38.46 (t, C-3), 45.99 (s, C-4), 56.67 (d, C-5), 21.35 (t, C-6), 39.18 (t, C-7), 147.72 (s, C-8), 56.52 (d, C-9), 40.58 (s, C-10), 26.23 (t, C-11), 36.23 (t, C-12), 31.39 (d, C-13), 43.89 (t, C-14), 173.23 (s, C15), 20.01 (q, C-16), 107.09 (t, C-17), 28.22 (q, C-18), 170.70 (s, C-19), 13.36 (q, C-20); Gly: 172.93 (s, C'-1), 41.29 (t, C'-2); 52.44 (q, OMe); ESI-MS: 430.2655. Calcd for $\left[\mathrm{C}_{23} \mathrm{H}_{37} \mathrm{NO}_{5} \mathrm{Na}^{+}\right]: 430.2570$.

Labd-8(17)-en-15,19-dioic acid, 15-valyl methyl ester amide (12). The diacid (215 mg, $0.64 \mathrm{mmol})$ was treated with DCC/DMAP and valine methyl ester.HCl to afford after the usual work-up and purification by Sephadex LH-20, the compound 12 as a colourless resin (34 $\mathrm{mg}, 0.076 \mathrm{mmol}, 12 \%$ yield, Rf: 0.40, PE:EtOAc 1:1). $[\alpha]_{D}^{20}:+20\left(c 0.74, \mathrm{CHCl}_{3}\right)$; IR $v_{\max }$ (film) 3295, 2956, 2932, 2868, 1752, 1720, 1692, 1641, 1545, 1210, 1170, $756 \mathrm{~cm}^{-1} ;{ }^{1} \mathrm{H}$ NMR $\left(\mathrm{CDCl}_{3}\right) \delta 0.52(3 \mathrm{H}, \mathrm{s}, \mathrm{H}-20), 0.85(3 \mathrm{H}$, d, $J=6.0 \mathrm{~Hz}, \mathrm{H}-16), 0.85$ (6H, d, $J=6.5 \mathrm{~Hz}$, Val H-4, H-5), 1.16 (3H, s, H-18), 3.66 (3H, s), 4.40 
(1H, s, H-17), 4.60 (1H, ddd, $J=13.6,8.8,4.8 \mathrm{~Hz}, \mathrm{Val} \mathrm{H}-2), 4.75(1 \mathrm{H}, \mathrm{s}, \mathrm{H}-17), 5.99(1 \mathrm{H}, \mathrm{d}, J=8$, $\mathrm{NH}) ;{ }^{13} \mathrm{C} \mathrm{NMR}\left(\mathrm{CDCl}_{3}\right): 38.92$ (t, C-1), 20.10 (t, C-2), 41.85 (t, C-3), 44.31 (s, C-4), 56.50 (d, C-5), 26.23 (t, C-6), 39.30 (t, C-7), 148.18 (s, C-8), 50.66 (d, C-9), 40.71 (s, C-10), 22.05 (t, C-11), 36.25 (t, C-12), 31.61 (d, C-13), 44.15 (t, C-14), 174.17 (s, C-15), 21.24 (q, C-16), 106.63 (t, C-17), 29.20 (q, C-18), 183.37 (s, C-19), 13.05 (q, C-20); Val: 172.83 (s, C'-1), 56.71 (d, C'-2), 31.61 (d, C'-3), 24.84 (q, C'-4), 23.00 (q, C'-5); 52.44 (q, OMe); ESI-MS: 472.6656. Calcd for $\left[\mathrm{C}_{26} \mathrm{H}_{43} \mathrm{NO}_{5} \mathrm{Na}^{+}\right]: 472.3039$.

Labd-8(17)-en-15,19-dioic acid, 15-leucyl methyl ester amide (13). The diacid (205 mg, $0.61 \mathrm{mmol})$ was treated with DCC/DMAP and leucine methyl ester.HCl to afford after the usual work-up and purification by $\mathrm{CC}$ on silica gel the compound 13 as colourless resin (62 $\mathrm{mg}, 0.134 \mathrm{mmol}, 22 \%$ yield, Rf: 0.26, PE:EtOAc 7:3). $[\alpha]_{D}^{20}:+16\left(c 0.63, \mathrm{CHCl}_{3}\right.$ ); IR $v_{\max }$ (film) 3303, 2952, 2928, 2864, 1748, 1716, 1645, 1541, 1437, 1202, $1162 \mathrm{~cm}^{-1} ;{ }^{1} \mathrm{H}$ NMR $\left(\mathrm{CDCl}_{3}\right) \delta 0.53(3 \mathrm{H}, \mathrm{s}, \mathrm{H}-20), 0.86(3 \mathrm{H}, \mathrm{d}, J=6.0$ Hz, H-16), 0.87 (6H, d, $J=6.0$ Hz, Leu H-5 and H-6), 1.17 (3H, s, H-18), 3.67 (3H, s), 4.40 (1H, s, H17), 4.61 (1H, ddd, $J=13.6,8.8,5.2 \mathrm{~Hz}$, Leu H-2), $4.75(1 \mathrm{H}, \mathrm{s}, \mathrm{H}-17), 5.93(1 \mathrm{H}, \mathrm{d}, J=8.4 \mathrm{~Hz}, \mathrm{NH})$; ${ }^{13} \mathrm{C} \mathrm{NMR}\left(\mathrm{CDCl}_{3}\right): 39.34$ (t, C-1), 20.13 (t, C-2), 38.22 (t, C-3), 44.33 (s, C-4), 56.74 (d, C-5), 21.28 (t, C-6), 38.94 (t, C-7), 148.23 (s, C-8), 56.53 (d, C-9), 40.74 (s, C-10), 26.26 (t, C-11), 36.23 (t, C-12), 31.65 (d, C-13), 44.22 (t, C-14), 172.73 (s, C-15), 23.02 (q, C-16), 106.66 (t, C-17), 29.22 (q, C-18), 183.05 (s, C-19), 12.99 (q, C-20); Leu: 174.10 (s, C'-1), 50.69 (d, C'-2), 41.92 (t, C'-3), 25.04 (d, C'4), 20.05 (q, C'-5), 22.08 (q, C'-6); 52.44 (q, OMe); ESI-MS: 486.3339. Calcd for $\left[\mathrm{C}_{27} \mathrm{H}_{45} \mathrm{NO}_{5} \mathrm{Na}^{+}\right]$: 486.3195 .

Labd-8(17)-en-15,19-dioic acid, 15-prolyl methyl ester amide (14). The diacid (174 mg, $0.518 \mathrm{mmol})$ was treated with oxalyl chloride and the acid chloride was reacted with proline methyl ester.HCl/TEA to afford after the usual work-up and purification by CC on RP-8 silica the compound $\mathbf{1 4}$ as colourless resin (22 mg, $0.049 \mathrm{mmol}, 10 \%$ yield, Rf: 0.15, PE:EtOAc 1:1). $[\alpha]_{D}^{20}:+4\left(c 0.022, \mathrm{CHCl}_{3}\right)$; IR $v_{\max }$ (film) 3390, 2956, 2872, 1792, 1752, 1641, 1441, 1413, 1198, 1170, 995, $764 \mathrm{~cm}^{-1} ;{ }^{1} \mathrm{H} \mathrm{NMR}\left(\mathrm{CDCl}_{3}\right)$ $\delta 0.60$ (3H, s, H-20), 0.93 (3H, d, $J=6.4 \mathrm{~Hz}, \mathrm{H}-16), 1.22$ (3H, s, H-18), 3.46 (1H, m, Pro H-5), 3.60 (1H, m, Pro H-5), 3.69 (3H, s), 4.45 (1H, m, Pro H-2), $4.47(1 \mathrm{H}, \mathrm{s}, \mathrm{H}-17), 4.80(1 \mathrm{H}, \mathrm{s}, \mathrm{H}-17) ;{ }^{13} \mathrm{C}$ NMR ( $\left.\mathrm{CDCl}_{3}\right): 39.21$ (t, C-1), 19.99 (t, C-2), 38.51 (t, C-3), 46.03 (s, C-4), 56.72 (d, C-5), 26.27 (t, C6), 38.91 (t, C-7), 147.83 (s, C-8), 56.55 (d, C-9), 40.62 (s, C-10), 21.44 (t, C-11), 36.52 (t, C-12), 31.11 (d, C-13), 41.59 (t, C-14), 171.83 (s, C-15), 20.26 (q, C-16), 107.19 (t, C-17), 28.27 (q, C-18), 173.29 (s, C-19), 13.55 (q, C-20); Pro: 173.15 (s, C-1'); 58.80 (d, C-2'); 29.27 (t, C-3'); 25.03 (t, C4'); 47.42 (t, C-5'); 52.29 (q, OMe); ESI-MS: 470.1352. Calcd for $\left[\mathrm{C}_{26} \mathrm{H}_{41} \mathrm{NO}_{5} \mathrm{Na}^{+}\right]$: 470.2882.

Labd-8(17)-en-15,19-dioic acid, 15-tryptophanyl methyl ester amide (15). The diacid (205 mg, $0.61 \mathrm{mmol}$ ) was treated with DCC/DMAP and tryptophane methyl ester.HCl to afford after the usual work-up and purification by $\mathrm{CC}$ on silica gel, the compound $\mathbf{1 5}$ as a colorless resin (58 $\mathrm{mg}$, 0.108 mmol, 18\% yield, Rf: 0.22, PE:EtOAc 1:1). $[\alpha]_{D}^{20}:+63$ (c $\left.0.032, \mathrm{CHCl}_{3}\right)$; IR $v_{\max }$ (film) 3406, 3311, 2944, 2848, 1796, 1736, 1649, 1457, 1214, 999, $744 \mathrm{~cm}^{-1} ;{ }^{1} \mathrm{H}$ NMR $\left(\mathrm{CDCl}_{3}\right) \delta 0.54(3 \mathrm{H}, \mathrm{s}, \mathrm{H}-$ 20), 0.79 (3H, d, $J=6.2 \mathrm{~Hz}, \mathrm{H}-16), 1.17 \mathrm{~s}(3 \mathrm{H}, \mathrm{s}, \mathrm{H}-18), 3.21$ (2H, m, Tript H-3), 3.60 (3H, s), 4.33 $(1 \mathrm{H}, \mathrm{s}, \mathrm{H}-17), 4.73(1 \mathrm{H}, \mathrm{s}, \mathrm{H}-17), 4.91(1 \mathrm{H}, \mathrm{m}$, Tript H-2), $5.90(1 \mathrm{H}, \mathrm{d}, J=8.0 \mathrm{~Hz}, \mathrm{NH}), 6.89(1 \mathrm{H}, \mathrm{br}$ s, Tript H-5), 7.00 (1H, dd, $J=7.4,7.4$ Hz, Tript H-8), 7.10 (1H, dd, $J=7.4,7.3 \mathrm{~Hz}$, Tript H-9), 7.27 
$(1 \mathrm{H}, \mathrm{d}, J=8.0 \mathrm{~Hz}$, Tript H-7), $7.44(1 \mathrm{H}, \mathrm{d}, J=7.8 \mathrm{~Hz}$, Tript H-10), $8.28(1 \mathrm{H}, \mathrm{s}, \mathrm{NH}), 10.78(1 \mathrm{H}, \mathrm{s}$, $\mathrm{NH}) ;{ }^{13} \mathrm{C}$ NMR $\left(\mathrm{CDCl}_{3}\right): 39.25$ (t, C-1), 20.02 (t, C-2), 38.54 (t, C-3), 46.07 (s, C-4), 56.75 (d, C-5), 26.31 (t, C-6), 38.93 (t, C-7), 147.80 (s, C-8), 56.61 (d, C-9), 40.66 (s, C-10), 21.43 (t, C-11), 36.31 (t, C-12), 31.56 (d, C-13), 44.24 (t, C-14), 172.48 (s, C-15), 20.01 (q, C-16), 107.21 (t, C-17), 28.29 (q, C-18), 173.32 (s, C-19), 13.59 (q, C-20); Trypt: 172.75 (s, C-1'); 52.98 (d, C-2'); 27.95 (t, C-3'); 110.46 (s, C-4'); 122.84 (d, C-5'); 136.33 (s, C-6'); 111.47 (d, C-7'); 119.94 (d, C-8'); 122.51 (d, C$\left.9^{\prime}\right) ; 118.82$ (d, C-10'); 127.96 (s, C-11'); 52.51 (q, OMe); ESI-MS: 559.3301. Calcd for $\left[\mathrm{C}_{32} \mathrm{H}_{44} \mathrm{~N}_{2} \mathrm{O}_{5} \mathrm{Na}^{+}\right]: 559.3250$.

Labd-8(17)-en-15,19-dioic acid, 15,19-diglycyl methyl ester amide (16). The diacid (197 mg, $0.586 \mathrm{mmol}$ ) was treated with oxalyl chloride and the acid chloride was reacted with glycine methyl ester.HCl/TEA to afford after the usual work-up and purification by gel permeation on Sephadex LH20, the compound 16 as colorless resin (131 mg, $0.274 \mathrm{mmol}, 47 \%$ yield, Rf: 0.29, PE:EtOAc 1:1). $[\alpha]_{D}^{20}:+30\left(c 0.13, \mathrm{CHCl}_{3}\right)$; IR $v_{\max }\left(\right.$ film) $3378,2948,2932,1756,1649,1529,1206,1178,748 \mathrm{~cm}^{-1}$; ${ }^{1} \mathrm{H}$ NMR $\left(\mathrm{CDCl}_{3}\right) \delta 0.49(3 \mathrm{H}, \mathrm{s}, \mathrm{H}-20), 0.86(3 \mathrm{H}, \mathrm{d}, J=6.0 \mathrm{~Hz}, \mathrm{H}-16), 1.13(3 \mathrm{H}, \mathrm{s}, \mathrm{H}-18), 3.67(3 \mathrm{H}$, s), $3.68(3 \mathrm{H}, \mathrm{s}), 3.92$ (2H, m, Gly H-2), 3.97 (2H, m, Gly H-2), 4.40 (1H, s, H-17), 4.75 (1H, s, H-17), $6.19\left(1 \mathrm{H}\right.$, br s, NH), $6.27\left(1 \mathrm{H}\right.$, br s, NH); ${ }^{13} \mathrm{C} \mathrm{NMR}\left(\mathrm{CDCl}_{3}\right): 39.03$ (t, C-1), 20.22 (t, C-2), 38.41 (t, C3), 44.27 (s, C-4), 56.85 (d, C-5), 21.33 (t, C-6), 39.53 (t, C-7), 147.97 (s, C-8), 56.55 (d, C-9), 40.55 (s, C-10), 26.67 (t, C-11), 36.23 (t, C-12), 31.53 (d, C-13), 43.78 (t, C-14), 173.29 (s, C-15), 19.92 (q, C-16), 106.73 (t, C-17), 30.10 (q, C-18), 177.17 (s, C-19), 12.88 (q, C-20); Gly: 170.88, 170.63 (s, C'1), 41.43, 41.28 (t, C'-2); 52.40 (q, 2 C, OMe); ESI-MS: 501.2332. Calcd. for $\left[\mathrm{C}_{26} \mathrm{H}_{42} \mathrm{~N}_{2} \mathrm{O}_{6} \mathrm{Na}^{+}\right]$: 501.2941 .

Labd-8(17)-en-15,19-dioic acid, 15,19-divalyl methyl ester amide (17). Some $0.392 \mathrm{~g}$ of the diacid $(1.17 \mathrm{mmol})$ were treated with oxalyl chloride to obtain the corresponding acid chloride who was treated with valine methyl ester.HCl/TEA to afford the diamide $\mathbf{1 7}$ as a colorless resin $(0.293 \mathrm{~g}$, 0.521 mmol, 44\% yield, Rf: 0.52, PE:EtOAc 6:4). $[\alpha]_{D}^{20}:+34$ (c 2.93, $\mathrm{CHCl}_{3}$ ); IR $v_{\max }$ (film) 3326, 2964, 1740, 1645, 1505, 1210, $768 \mathrm{~cm}^{-1} ;{ }^{1} \mathrm{H}$ NMR $\left(\mathrm{CDCl}_{3}\right) \delta 0.59(3 \mathrm{H}, \mathrm{s}, \mathrm{H}-20), 0.89(3 \mathrm{H}, \mathrm{d}$, $J=6.4 \mathrm{~Hz}, \mathrm{H}-16), 0.90(3 \mathrm{H}, \mathrm{d}, J=6.9 \mathrm{~Hz}), 0.91(6 \mathrm{H}, \mathrm{d}, J=6.9 \mathrm{~Hz}), 0.92(3 \mathrm{H}, \mathrm{d}, J=6.9 \mathrm{~Hz})(\mathrm{Val} \mathrm{H}-$ 4, H-5), 1.21 (3H, s, H-18), 3.73 (3H, s), $3.74(3 \mathrm{H}, \mathrm{s}), 4.48(1 \mathrm{H}, \mathrm{s}, \mathrm{H}-17), 4.49$ (1H, dd, $J=8.4$, $4.0 \mathrm{~Hz}$, Val H-2), $4.58(1 \mathrm{H}, \mathrm{dd}, J=8.8,4.8 \mathrm{~Hz}, \mathrm{Val} \mathrm{H}-2), 4.84(1 \mathrm{H}, \mathrm{s}, \mathrm{H}-17), 6.05(1 \mathrm{H}, \mathrm{d}, J=8.4 \mathrm{~Hz}$, $\mathrm{NH}), 6.13(1 \mathrm{H}, \mathrm{d}, J=8.4 \mathrm{~Hz}, \mathrm{NH})$; ESI-MS: 585.4012. Calcd for $\left[\mathrm{C}_{32} \mathrm{H}_{54} \mathrm{~N}_{2} \mathrm{O}_{6} \mathrm{Na}^{+}\right]: 585.3982$.

Labd-8(17)-en-15,19-dioic acid, 15,19-dileucyl methyl ester amide (18). Treatment of the diacid $(0.378 \mathrm{~g}, 1.13 \mathrm{mmol})$ with oxalyl chloride and leucine methyl ester.HCl/TEA afforded the diamide $\mathbf{1 8}$ as colourless resin $\left(229.5 \mathrm{mg}, 0.39 \mathrm{mmol}, 34.5 \%\right.$ yield, Rf 0.50, PE:EtOAc 6:4). $[\alpha]_{D}^{20}:+9.94(c$ $\left.0.503, \mathrm{CHCl}_{3}\right)$; IR $v_{\max }$ (film) 3318, 2956, 2872, 1748, 1641, 1521, 1202, $768 \mathrm{~cm}^{-1} ;{ }^{1} \mathrm{H} \mathrm{NMR}\left(\mathrm{CDCl}_{3}\right)$ $\delta 0.51(3 \mathrm{H}, \mathrm{s}, \mathrm{H}-20), 0.86(3 \mathrm{H}, \mathrm{d}, J=6.2 \mathrm{~Hz}, \mathrm{H}-16), 0.88(6 \mathrm{H}, \mathrm{d}, J=6.1 \mathrm{~Hz}, \mathrm{Leu} \mathrm{H}-5$ and H-6), 1.12 $(3 \mathrm{H}, \mathrm{s}, \mathrm{H}-18), 3.65(3 \mathrm{H}, \mathrm{s}), 3.66(3 \mathrm{H}, \mathrm{s}), 4.41(1 \mathrm{H}, \mathrm{s}, \mathrm{H}-17), 4.51(1 \mathrm{H}, \mathrm{ddd}, J=13.2,8.4,4.8 \mathrm{~Hz}$, Leu $\mathrm{H}-2), 4.58(1 \mathrm{H}, \mathrm{ddd}, J=13.2,8.8,4.8 \mathrm{~Hz}$, Leu H-2), $4.77(1 \mathrm{H}, \mathrm{s}, \mathrm{H}-17), 5.87(1 \mathrm{H}, \mathrm{d}, J=8.4 \mathrm{~Hz}, \mathrm{NH})$, $5.90(1 \mathrm{H}, \mathrm{d}, J=8.0 \mathrm{~Hz}, \mathrm{NH}) ;{ }^{13} \mathrm{C} \mathrm{NMR}\left(\mathrm{CDCl}_{3}\right): 39.62$ (t, C-1), 20.37 (t, C-2), 38.63 (t, C-3), 44.32 (s, C-4), 56.99 (d, C-5), 21.35 (t, C-6), 39.11 (t, C-7), 147.97 (s, C-8), 56.66 (d, C-9), 40.61 (s, C-10), 
26.66 (t, C-11), 36.26 (t, C-12), 31.60 (d, C-13), 44.18 (t, C-14), 172.51 (s, C-15), 20.02 (q, C-16), 106.79 (t, C-17), 30.12 (q, C-18), 176.62 (s, C-19), 13.03 (q, C-20); Leu: 173.84 (s, 2 C, C'-1); 50.72, 50.63 (d, C'-2), 41.84, 41.47 (t, C'-3), 25.16, 24.98 (d, C'-4), 22.02 (q, 2 C, C'-5), 22.97 (q, 2 C,. C'6); 52.31, 52.23 (q, OMe); ESI-MS: 613.3647. Calcd for $\left[\mathrm{C}_{34} \mathrm{H}_{58} \mathrm{~N}_{2} \mathrm{O}_{6} \mathrm{Na}^{+}\right]$: 613.4193.

Labd-8(17)-en-15,19-dioic acid, 15,19-diprolyl methyl ester amide (19). Some $0.400 \mathrm{~g}$ (1.19 mmol) of the diacid was treated with oxalyl chloride to afford the corresponding acid chloride, which was treated with proline methyl ester.HCl/TEA to obtain the diamide 19 as colourless resin $(256 \mathrm{mg}, 0.46 \mathrm{mmol}$, 38.6\% yield, Rf: 0.32, PE:EtOAc 1:1). [ $\alpha]_{D}^{20}:-48.4$ (c 2.34, $\mathrm{CHCl}_{3}$ ); IR $v_{\max }$ (film) 2952, 2880, 1744, $1633,1433,1198,1166,756 \mathrm{~cm}^{-1} ;{ }^{1} \mathrm{H}$ NMR $\left(\mathrm{CDCl}_{3}\right) \delta 0.53(3 \mathrm{H}, \mathrm{s}, \mathrm{H}-20), 0.87(3 \mathrm{H}, \mathrm{d}, J=6.4 \mathrm{~Hz}, \mathrm{H}-$ 16), 1.15 (3H, s, H-18), 3.40 (1H, m, Pro H-5), 3.52 (3H, s), 3.56 (1H, m, Pro H-5), 3.62 (3H, s), 4.39 (1H, s, H-17), 4.41 (1H, m, Pro H-2), 4.73 (1H, s, H-17); ${ }^{13} \mathrm{C}$ NMR $\left(\mathrm{CDCl}_{3}\right): 40.09$ (t, C-1), 20.86 (t, C-2), 38.85 (t, C-3), 46.25 (s, C-4), 60.56 (d, C-5), 26.79 (t, C-6), 39.44 (t, C-7), 148.48 (s, C-8), 57.43 (d, C-9), 41.02 (s, C-10), 21.37 (t, C-11), 36.55 (t, C-12), 31.08 (d, C-13), 41.44 (t, C-14), 171.78 (s, C-15), 20.17 (q, C-16), 106.03 (t, C-17), 27.11 (q, C-18), 176.01 (s, C-19), 14.94 (q, C-20); Pro: 173.47, 172.96 (s, C-1'); 61.75, 58.66 (d, C-2'); 29.26 (t, C-3'); 24.86 (t, C-4'); 48.23, 47.27 (t, C-5'); 52.30, 51.32 (q, OMe); ESI-MS: 581.3036. Calcd for $\left[\mathrm{C}_{32} \mathrm{H}_{50} \mathrm{~N}_{2} \mathrm{O}_{6} \mathrm{Na}^{+}\right]: 581.3566$.

Labd-8(17)-en-15,19-dioic acid, 15,19-ditryptophanyl ethyl ester amide (20). Some $0.170 \mathrm{~g}$ of the diacid $(0.506 \mathrm{mmol})$ was treated with oxalyl chloride to afford the corresponding acid chloride, which was reacted with tryptophane ethyl ester.HCl/TEA to obtain the diamide 20 (161 mg, $0.211 \mathrm{mmol}$, 42\% yield, Rf: 0.56, PE:EtOAc 1:1). $[\alpha]_{D}^{20}:+46$ (c 0.097, $\mathrm{CHCl}_{3}$ ); IR $v_{\max }$ (film) 3414, 3311, 3932, 2868, 1736, 1637, 1509, 1461, 1214, $744 \mathrm{~cm}^{-1} ;{ }^{1} \mathrm{H}$ NMR $\left(\mathrm{CDCl}_{3}\right) \delta 0.39(3 \mathrm{H}, \mathrm{s}, \mathrm{H}-20), 0.83(3 \mathrm{H}, \mathrm{d}$, $J=6.2 \mathrm{~Hz}, \mathrm{H}-16), 1.02(3 \mathrm{H}, \mathrm{s}, \mathrm{H}-18), 1.15(3 \mathrm{H}, \mathrm{q}, J=6.6 \mathrm{~Hz}$, Ethyl), 1.17 (3H, q, $J=6.6 \mathrm{~Hz}$, Ethyl), 3.26 (4 H, m, Tript H-3), 4.09 (4H, m, Ethyl), 4.37 (1H, s, H-17), 4.74 (1H, s, H-17), 4.77 (1H, m, Tript H-2), 4.93 (1H, m, Tript H-2), $6.00(1 \mathrm{H}, \mathrm{d}, J=7.9 \mathrm{~Hz}$, Tript NH), $6.05(1 \mathrm{H}, \mathrm{d}, J=6.8 \mathrm{~Hz}$, Tript NH), $6.93(1 \mathrm{H}, \mathrm{d}, J=1.9 \mathrm{~Hz}$, Tript H-5), $6.95(1 \mathrm{H}, \mathrm{d}, J=1.9 \mathrm{~Hz}$, Tript H-5), 7.09 (2H, dd, $J=7.8$, 7.1 Hz, Tript H-8), 7.16 (2H, dd, $J=7.5,7.2 \mathrm{~Hz}$, Tript H-9), 7.32 (2H, d, $J=8.1 \mathrm{~Hz}$, Tript H-7), 7.51 $\left(1 \mathrm{H}, \mathrm{d}, J=8.1 \mathrm{~Hz}\right.$, Tript H-10), $7.54\left(1 \mathrm{H}, \mathrm{d}, J=8.2 \mathrm{~Hz}\right.$, Tript H-10), $8.59\left(2 \mathrm{H}\right.$, brs, NH); ${ }^{13} \mathrm{C}$ NMR $\left(\mathrm{CDCl}_{3}\right): 39.51$ (t, C-1), 20.01 (t, C-2), 38.49 (t, C-3), 44.22 (s, C-4), 56.77 (d, C-5), 26.53 (t, C-6), 39.03 (t, C-7), 148.01 (s, C-8), 56.52 (d, C-9), 40.52 (s, C-10), 21.31 (t, C-11), 36.27 (t, C-12), 31.50 (d, C-13), 44.09 (t, C-14), 172.27 (s, C-15), 20.01 (q, C-16), 106.76 (t, C-17), 30.01 (q, C-18), 176.88 (s, C-19), 12.94 (q, C-20); Trypt: 172.66, 172.47 (s, C-1'); 53.28, 53.09 (d, C-2'); 27.87, 27.51 (t, C3'); 110.05 (s, 2 C, C-4'); 122.99, 122.91 (d, C-5'); 136.33 (s, 2 C, C-6'); 111.52, 111.41 (d, C-7'); 119.66 (d, 2 C, C-8'); 122.34, 122.24 (d, C-9'); 118.62, 118.50 (d, C-10'); 127.88, 127.63 (s, C-11'); Ethyl: 61.60, 61.48 (t; ethyl); 14.21, 14.14 (q, Ethyl); ESI-MS: 787.9840. Calc for $\left[\mathrm{C}_{46} \mathrm{H}_{60} \mathrm{~N}_{4} \mathrm{O}_{6} \mathrm{Na}^{+}\right]$: 787.4410 .

\subsection{Ethanol/HCl-induced ulcer model in mice}

The gastroprotective activity of the compounds was determined in the ethanol/HCl-induced lesion model in mice $[14,15,20,21]$. The purity of the tested compounds was higher than $95 \%$ by NMR 
analysis. Male Swiss albino mice weighing $30 \pm 3 \mathrm{~g}$ were used. Animals were fed on certified Champion diet with free access to water under standard conditions of $12 \mathrm{~h}$ dark-light period, $50 \%$ relative humidity and $22{ }^{\circ} \mathrm{C}$ room temperature. Mice were randomly distributed into groups of eight animals each and fasted for $24 \mathrm{~h}$ with free access to water previous to the experiment. Fasting prior to ulcerogenic assays was used because $0.2 \mathrm{~mL}$ of the reference antisecretory drug lansoprazole (2-[[[3methyl-4-(2,2,2-trifluroethoxy)-2-pyridyl]methyl]sulfinyl]benzimidazole) and the amides were administered orally. To keep the animal number to a minimum, dose-response studies were performed with the leucine derivatives $\mathbf{1 3}$ and $\mathbf{1 8}$ to set the conditions for single-dose comparison of gastroprotective effect. For the selected parent compounds, three doses were used: 25, 50 and $100 \mathrm{mg} / \mathrm{kg}$. Fifty min after oral administration of the compounds, lansoprazole $(20 \mathrm{mg} / \mathrm{kg})$ or the vehicle $(12 \%$ Tween $80,10 \mathrm{~mL} / \mathrm{kg})$, all groups were orally treated with $0.2 \mathrm{~mL}$ of a solution containing $60 \%$ ethanol/ $/ 0.3 \mathrm{M} \mathrm{HCl}$ (ethanol/ $\mathrm{HCl}$ ) for gastric lesion induction. Animals were sacrificed by cervical dislocation $1 \mathrm{~h}$ after the administration of ethanol/ $\mathrm{HCl}$, and the stomachs excised and inflated by injection of $5 \%$ formalin $(1 \mathrm{~mL})$. The ulcerated stomachs were fixed in $5 \%$ formalin for $30 \mathrm{~min}$ and opened along the greater curvature. The length $(\mathrm{mm})$ of each lesion was measured, and the lesion index expressed as the sum of the length of all lesions [14,15,20,21]. For comparison purposes, the products 1-5, 7-12, 14-16, 19 and 20 were assessed at a single oral dose of $25 \mathrm{mg} / \mathrm{kg}$. The protocols were approved by the Universidad de Talca Institutional Animal Care and Use Committee, which follows the recommendations of the Canadian Council on Animal Care [23]. Tween 80 and lansoprazole ( $>98 \%$ purity by HPLC) were purchased from Sigma Chemical Co.

\subsection{Cell culture}

\subsubsection{MRC-5 and Hep G2 cells}

Human normal lung fibroblasts MRC-5 (ATCC CCL-171) and Hep G2 cells (ATCC HB-8065) were grown as monolayers in minimum essential Eagle's medium (MEM), with Earle's salts, $2.0 \mathrm{mM}$ L-glutamine (Sigma Chemical Co.) and $2.0 \mathrm{~g} / \mathrm{L}$ sodium bicarbonate (Sigma Chemical Co.), supplemented with 10\% heat-inactivated fetal bovine serum (FBS), $100 \mathrm{IU} / \mathrm{mL}$ penicillin and $100 \mu \mathrm{g} / \mathrm{mL}$ streptomycin in a humidified incubator with $5 \% \mathrm{CO}_{2}$ in air at $37^{\circ} \mathrm{C}$. Cell passage was maintained between 10 and 16 for MRC-5 and between 79 and 82 for Hep G2 cells. The medium was changed every 2 days. Culture media, antibiotics and FBS were obtained from Invitrogen Corp.

\subsubsection{AGS cells}

Human gastric adenocarcinoma cells AGS (ATCC CRL-1739) were grown as monolayers in Ham F-12 medium containing $1.0 \mathrm{mM}$ L-glutamine and $1.5 \mathrm{~g} / \mathrm{L}$ sodium bicarbonate, supplemented with $10 \%$ heat-inactivated FBS, $100 \mathrm{IU} / \mathrm{mL}$ penicillin and $100 \mu \mathrm{g} / \mathrm{mL}$ streptomycin in a humidified incubator with $5 \% \mathrm{CO}_{2}$ in air at $37{ }^{\circ} \mathrm{C}$. The cell passage was maintained between 42 and 48 . The medium was changed every 2 days. 


\subsection{Cytotoxicity assay}

Confluent cultures of MRC-5, AGS and Hep G2 cells were treated with medium containing the compounds at concentrations ranging from 0 up to $1000 \mu \mathrm{M}$. The products were first dissolved in DMSO and then in the corresponding culture medium supplemented with $2 \% \mathrm{FBS}$. The final content of DMSO in the test medium and controls was $1 \%$. Cells were exposed for $24 \mathrm{~h}$ to the assayed compounds. Untreated cells served as controls. Each concentration was tested in quadruplicate together with the control and repeated three times in separate experiments. At the end of the incubation, the neutral red uptake (NRU) assay was carried out to determine cell viability [20,21]. To calculate the $\mathrm{IC}_{50}$ values (concentration that produces a $50 \%$ inhibitory effect on the evaluated parameter) the results were transformed to percentage of controls and the $\mathrm{IC}_{50}$ value was graphically obtained from the dose-response curves.

\subsection{Statistical analysis}

Results were expressed as the mean \pm SD. In all experiments, statistical differences between several treatments and their respective control were determined by one-way analysis of variance (ANOVA) and when the F value was significant, post hoc differences were determined by the Dunnett's multiple comparison test. The level of significance was set at $\mathrm{P}<0.05$. All statistical analyses were performed using the software Statistica 5.1 (StatSoft, Inc.) and Statistical Package S-Plus 2000.

\section{Conclusions}

In summary, the new compounds prepared were more active as gastroprotective agents than the C-19 monoamides prepared from imbricatolic acid, 15-acetoxyimbricatolic acid and the corresponding 8(9)-en isomers [21]. Relevant gastric lesion-preventing effects with very low cytotoxicity were found at a single oral dose of $25 \mathrm{mg} / \mathrm{kg}$ for some of the compounds. Further studies are needed to fully reveal the potential of labdane diterpenes as templates for the synthesis of new gastroprotective drugs.

\section{Acknowledgements}

This work was supported by FONDECYT Project $\mathrm{N}^{\circ} 1085306$ and Programa de Investigación en Productos Bioactivos, Universidad de Talca. The skillful technical work of Irene Manriquez is gratefully acknowledged.

\section{References}

1. Farina, C.; Pinza, M.; Pifferi, G. Synthesis and anti-ulcer activity of new derivatives of glycyrrhetic, oleanolic and ursolic acids. Farmaco 1998, 53, 22-32.

2. Onoda, Y.; Magaribuchi, T.; Tamaki, H. Effects of the new anti-ulcer agent 12sulfodehydroabietic acid monosodium salt on duodenal alkaline secretion in rats. Arzneimittelforschung 1990, 40, 576-578. 
3. Onoda, Y.; Takido, M.; Magaribuchi, T.; Tamaki, H. Effects of 12-sulfodehydroabietic acid monosodium salt (TA-2711), a new anti-ulcer agent, on gastric mucosal lesions induced by necrotizing agents and gastric mucosal defensive factors in rats. Jpn. J. Pharmacol. 1990, 52, 631-638.

4. Pearson, J.P.; Roberts, N.B. Mucosal protective effects of ecabet sodium: pepsin inhibition and interaction with mucus. Clin. Sci. 2001, 100, 411-417.

5. Wada, H.; Yodato, S.; Kawamori, M.; Morikawa, T.; Nakai, H.; Takeda, M.; Saito, S.; Onoda, Y.; Tamaki, H. Antiulcer activity of dehydroabietic acid derivatives. Chem. Pharm. Bull. 1985, 33, 1472-1487.

6. Hiruma-Lima, C.A.; Spadari-Bratfisch, R.C.; Grassi-Kassisse, D.M.; Brito, A.R. Antiulcerogenic mechanisms of dehydrocrotonin, a diterpene lactone obtained from Croton cajucara. Planta Med. 1999, 65, 325-330.

7. Hiruma-Lima, C.A.; Gracioso, J.D.; Toma, W.; de Paula, A.C.; de Almeida, A.B.; Brasil, D.D.; Muller, A.H.; Brito, A.R. Evaluation of the gastroprotective activity of cordatin, a diterpene isolated from Aparisthmium cordatum (Euphorbiaceae). Biol. Pharm. Bull. 2000, 23, 1465-1469.

8. Hiruma-Lima, C.A.; Gracioso, J.S.; Toma, W.; Almeida, A.B.; Paula, A.C.; Brasil, D.S.B.; Muller, A.H.; Souza-Brito, A.R.M. Gastroprotective effect of aparisthman, a diterpene isolated from Aparisthmium cordatum, on experimental gastric ulcer models in rats and mice. Phytomedicine 2001, 8, 94-100.

9. Rodríguez, J.A.; Bustamante, C.; Astudillo, L.; Schmeda-Hirschmann, G. Antiulcer activity of dehydroabietic acid derivatives. J. Pharm. Pharmacol. 2002, 54, 399-404.

10. Rodríguez, J.A.; Astudillo, L.; Schmeda-Hirschmann, G. Oleanolic acid promotes healing of acetic acid-induced chronic gastric ulcers in rats. Pharmacol. Res. 2003, 48, 291-294.

11. Rodríguez, J.A.; Theoduloz, C.; Sánchez, M.; Razmilic, I.; Schmeda-Hirschmann, G. Gastroprotective and ulcer-healing effect of new solidagenone derivatives in human cell cultures. Life Sci. 2005, 77, 2193-2205.

12. Rodríguez, J.A.; Theoduloz, C.; Sánchez, M.; Yáñez, T.; Razmilic, I.; Schmeda-Hirschmann, G. Gastroprotective activity of a new semi-synthetic solidagenone derivative in mice. J. Pharm. Pharmacol. 2005, 57, 265-271.

13. Schmeda-Hirschmann, G.; Rodríguez, J.A.; Astudillo, L. Gastroprotective activity of the diterpene solidagenone and its derivatives on experimentally induced gastric lesions in mice. $J$. Ethnopharmacol. 2002, 81, 111-115.

14. Schmeda-Hirschmann, G.; Astudillo, L.; Sepúlveda, B.; Rodríguez, J.A.; Theoduloz, C.; Yáñez, T.; Palenzuela, J.A. Gastroprotective effect and cytotoxicity of natural and semisynthetic labdane diterpenes from Araucaria araucana. Z. Naturforsch. 2005, 60c, 511-522.

15. Schmeda-Hirschmann, G.; Astudillo, L.; Rodríguez, J.A.; Theoduloz, C.; Yáñez, T. Gastroprotective effect of the Mapuche crude drug Araucaria araucana resin and its main constituents. J. Ethnopharmacol. 2005, 101, 271-276.

16. Pertino, M.; Schmeda-Hirschmann, G.; Rodríguez, J.A.; Theoduloz, C. Gastroprotective effect and cytotoxicity of terpenes from the Paraguayan crude drug "yagua rova" (Jatropha isabelli). J. Ethnopharmacol. 2007, 111, 553-559. 
17. Pertino, M.; Schmeda-Hirschmann, G.; Rodríguez, J.A.; Theoduloz, C. Gastroprotective effect and cytotoxicity of semisynthetic jatropholone derivatives. Planta Med. 2007, 73, 1095-1100.

18. Luo, J.; Nordenvall, C.; Nyren, O.; Adami, H.O.; Permert, J.; Ye, W. The risk of pancreatic cancer in patients with gastric or duodenal ulcer disease. Int. J. Cancer 2007, 120, 368-372.

19. Bahmanyar, S.; Ye, W.; Dickman, P.W.; Nyren, O. Long-term risk of gastric cancer by subsite in operated and unoperated patients hospitalized for peptic ulcer. Am. J. Gastroenterol. 2007, 102, 1185-1191.

20. Izquierdo, R.; Astudillo, L.; Rodríguez, J.A.; Theoduloz, C.; Palenzuela, J.A.; SchmedaHirschmann, G. Gastroprotective effect and cytotoxicity of labdenamides. Planta Med. 2007, 73, 310-317.

21. Schmeda-Hirschmann, G.; Rodriguez, J.; Theoduloz, C.; Yañez, T.; Valderrama, J. Gastroprotective effect and cytotoxicity of labdeneamides with amino acids. Planta Med. 2010, in press.

22. Yu, D.; Sakurai, Y.; Chen, Ch.-H.; Chang, F.-R.; Huang, L.; Kashiwada, Y.; Li, K.-H. Anti-AIDS Agents 69. Moronic acid and other triterpene derivatives as novel potent anti-HIV agents. J. Med. Chem. 2006, 49, 5462-5469.

23. Olfert, E.D.; Cross, B.M.; McWilliam, A.A. Guide to the Care and Use of Experimental Animals. Canadian Council on Animal Care; Canadian Council on Animal Care: Ottawa, Canada, 1993; Volume 1, pp. 213-214.

Sample Availability: Samples of the compounds junicedric acid, junicedric acid 8(9)-en isomer and imbricatolic acid are available from the authors.

(C) 2010 by the authors; licensee MDPI, Basel, Switzerland. This article is an open access article distributed under the terms and conditions of the Creative Commons Attribution license (http://creativecommons.org/licenses/by/3.0/). 